\title{
Attenuation of Oxidative Stress of Erythrocytes by Plant-Derived Flavonoids, Orientin and Luteolin
}

\author{
Fang An, ${ }^{1}$ Shulin Wang, ${ }^{2}$ Danhua Yuan, ${ }^{2}$ Yuewen Gong, ${ }^{3}$ and Shuhua Wang ${ }^{2}$ \\ ${ }^{1}$ Graduate Faculty, Hebei North University, Zhangjiakou, Hebei 075000, China \\ ${ }^{2}$ Department of Pharmacy, Hebei North University, Zhangjiakou, Hebei 075000, China \\ ${ }^{3}$ Faculty of Pharmacy, University of Manitoba, 750 McDermot Avenue, Winnipeg, MB, Canada R3E 0T5
}

Correspondence should be addressed to Shuhua Wang; wangshyh@126.com

Received 2 September 2015; Revised 7 January 2016; Accepted 11 January 2016

Academic Editor: G. K. Jayaprakasha

Copyright (C) 2016 Fang An et al. This is an open access article distributed under the Creative Commons Attribution License, which permits unrestricted use, distribution, and reproduction in any medium, provided the original work is properly cited.

Erythrocytes are easy to be injured by oxidative stress in their lifespan. Although there are several chemicals such as vitamin $\mathrm{C}(\mathrm{VC})$ that would be able to reduce oxidative stress, natural herbal products still remain an interesting research area. The current study investigated the effects of two plant-derived flavonoids, orientin and luteolin, on erythrocytes and their possible mechanisms. This experiment was divided into nine groups, which were normal group, model group, VC control group, and treated groups with different doses of orientin and luteolin $(10,20$, and $40 \mu \mathrm{g} / \mathrm{mL})$, respectively. Hemolysis rate was determined by spectrophotometry. Antioxidative enzyme and products were evaluated by different methods. Erythrocyte cell surface and cellular structure were observed with scanning or transmission electron microscope, respectively. Oxidative stress induced significant increase in hemolysis rate of erythrocytes. Orientin or luteolin ameliorated hemolysis of erythrocytes in oxidative stress in a dose-dependent manner. Both orientin and luteolin reduced oxidative products and increased antioxidative enzyme activities. Moreover, orientin and luteolin attenuated oxidative stress induced damage of erythrocyte cell surface morphology and cellular structure. In conclusion, orientin and luteolin could protect human erythrocytes from oxidative damage by attenuating oxidative stress, protecting antioxidative enzyme activities, and preserving integrity of erythrocyte structure.

\section{Introduction}

Erythrocytes are the most abundant visible components in the blood system. They usually have a lifespan of 120 days [1]. Reduced number of erythrocytes can cause anemia and lack of oxygen [2]. Since erythrocytes do not contain the nuclei and mitochondria, oxidative stress usually causes membrane lipid peroxidation. Moreover, erythrocyte membranes are rich in unsaturated fats and hemoglobin contains more iron molecules [3]. Both of these are powerful catalysts of free radical reactions. So erythrocytes are sensitive to oxidative damage from reactive oxygen species (ROS) [4]. Therefore, eliminating excess free radicals could reduce membrane lipid peroxidation and protect erythrocytes from oxidative injury.

At present, there are few known drugs that can prevent erythrocytes from oxidative injury. But chemical components extracted from plants have shown some promising trend especially herbs used in China [5]. Flavonoid is one of the chemical components extracted from stem, leaf, and flower of a wide variety of plants, which has a broad spectrum of pharmacological activities and low toxicity $[6,7]$. For example, anthocyanins isolated from the red bayberry have been shown to decrease the production of ROS and prevent oxidative damage to islet cells [8].

From our previous studies, we have identified two flavonoids from our local herbal plant (Trollius chinensis Bunge). Orientin belongs to the family of flavonoid glycosides and luteolin belongs to the flavonoid aglycone class [9]. Both of them have been demonstrated to have antioxidative activity in ischemic myocardial disease [10-12]. Their roles in prevention of erythrocytes from oxidative stress are still not completely understood. The current study is to investigate whether orientin and luteolin can protect erythrocytes from oxidative injury and whether there are some differences between these two compounds with different chemical structure (Figure 1). 

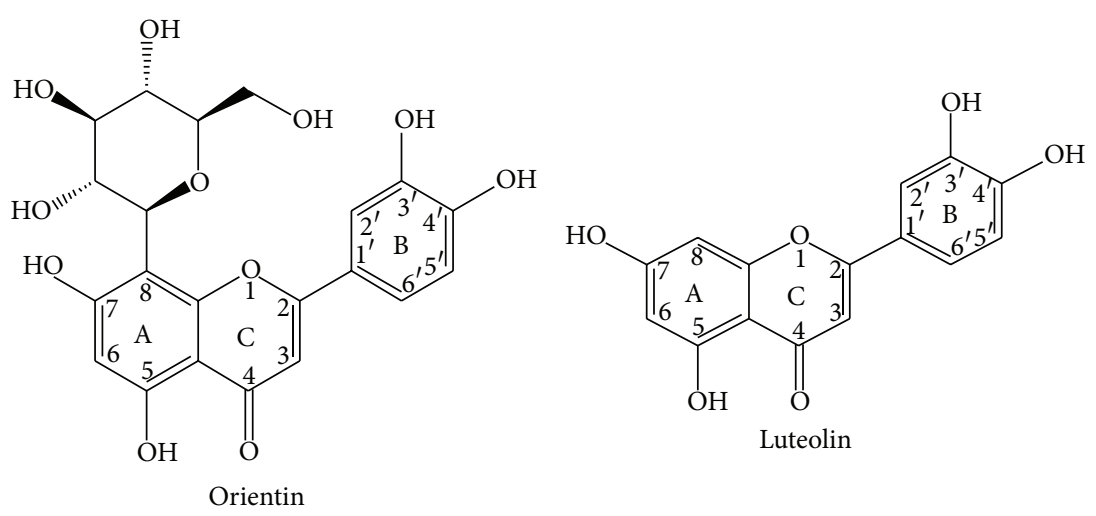

FIGURE 1: The structure of orientin and luteolin.

\section{Materials and Methods}

2.1. Preparation of Erythrocyte Suspension. Blood samples from healthy donors (Zhangjiakou Center Blood Stations) were centrifuged at $4^{\circ} \mathrm{C}, 2500 \mathrm{rpm}$ for $10 \mathrm{~min}$, to separate erythrocytes from the blood cells. The erythrocytes were washed three times with phosphate buffer saline (PBS) $(\mathrm{pH}$ 7.4 ) and resuspended at $2 \%$ in PBS.

2.2. Erythrocytes Oxidative Damage Model. 2\% erythrocyte suspension $(2.0 \mathrm{~mL})$ was added to $\mathrm{H}_{2} \mathrm{O}_{2}(2.0 \mathrm{~mL})$ at different concentrations and the final concentrations of $\mathrm{H}_{2} \mathrm{O}_{2}$ were $100,200,300,400$, and $500 \mathrm{mM}$, respectively. The group without $\mathrm{H}_{2} \mathrm{O}_{2}$ was the control group. Erythrocytes were taken from each tube every $30 \mathrm{~min}$ after the incubation at $37^{\circ} \mathrm{C}$, diluted 10 -fold with $0.9 \%$ saline or distilled water $\left(\mathrm{ddH}_{2} \mathrm{O}\right)$, and then centrifuged at $2500 \mathrm{rpm}$ for $10 \mathrm{~min}$ at $4^{\circ} \mathrm{C}$. The supernatants were collected to measure the absorbance value (UV-9100 UV-Vis Spectrophotometer, Beijing Lab Tech Instrument Co., Ltd., Beijing, China) at $412 \mathrm{~nm}$. The final hemolysis rate was determined by the final concentration of $\mathrm{H}_{2} \mathrm{O}_{2}$ and incubation time with the following formula: hemolysis rate $(\%)=A / A_{0} \times 100 \%(A$, absorbance value of sample diluted with saline; $A_{0}$, absorbance value of sample diluted with $\left.\mathrm{ddH}_{2} \mathrm{O}\right)[13,14]$.

2.3. Treatment of Erythrocytes with Different Drugs. Orientin (purity $\geq 98.6 \%$ ) and luteolin (purity $\geq 98.6 \%$ ) were purchased from Tianjin Party Ltd. and the purity was determined by high performance liquid chromatography (HPLC) and structure was confirmed by 1hydrogen-nuclear magnetic resonance $\left({ }^{1} \mathrm{H}-\mathrm{NMR}\right)$ [9]. Both orientin and luteolin were dissolved in dimethyl sulfoxide (DMSO) and then diluted with $\mathrm{CH}_{3} \mathrm{OH}$. The concentrations of DMSO and $\mathrm{CH}_{3} \mathrm{OH}$ were maintained at $0.01 \%$ and $0.8 \%$ of final concentrations, respectively. This experiment was divided into nine groups, which were normal group, model group, VC control group, and treated groups with different doses of orientin and luteolin $(10,20$, and $40 \mu \mathrm{g} / \mathrm{mL})$, respectively. For normal group and model group, erythrocytes were incubated with $100 \mu \mathrm{L}$ solution of DMSO and $\mathrm{CH}_{3} \mathrm{OH}$, and erythrocytes were incubated with same dose of different drugs in other groups. Pretreatment of erythrocytes of each group was incubated at $37^{\circ} \mathrm{C}$ for 30 minutes. At the end of incubation, $\mathrm{H}_{2} \mathrm{O}_{2}$ was added to the solution in model or each treatment group and $0.9 \%$ saline was added to the normal group. Erythrocytes were then incubated at $37^{\circ} \mathrm{C}$ for 1.5 hours and the final hemolysis rate was calculated as indicated above.

2.4. Determination of Oxidative Stress in Erythrocytes. Oxidative stress in erythrocytes was determined by measuring enzymatic levels of superoxide dismutase (SOD), catalase (CAT), glutathione peroxidase (GSH-Px), and adenosine trip phosphate system (ATPase). Moreover, ROS and malondialdehyde (MDA) were also measured. After establishment of erythrocyte oxidative damage model, the concentrations of the protein were measured by Bradford protein assay kit from Beyotime Institute of Biotechnology (Shanghai, China). Cytoplasmic proteins were employed for determination of activities of SOD, CAT, GSH-Px, and ATPase. MDA in erythrocyte cytoplasm was extracted using the methods of kits. All these parameters were measured according to the instructions of the available kits from Nanjing Jiancheng Bioengineering Institute (Nanjing, China, with batch numbers: 20120417 for total SOD, 20120419 for CAT, 20120417 for GSHPx, 20120420 for ATP, and 20120417 for MDA, resp.).

In the paper, the nonpolar 2,7-dichlorofluorescin diacetate (DCFH-DA) was used as a probe to investigate the level of ROS. DCFH-DA has no fluorescence itself; however, it could pass through the cell membrane freely and then was hydrolyzed by cellular esterases into DCFH. The intracellular DCFH could be easily oxidized by ROS into fluorescent dichlorofluorescein (DCH). Thus the ROS generation would be measured by the determination of DCF fluorescence intensity. At the end of treatment, $2 \%$ erythrocyte suspension was incubated with $10 \mathrm{mM} \mathrm{DCFH}-\mathrm{DA}$ at $37^{\circ} \mathrm{C}$ for $30 \mathrm{~min}$ and then washed twice with PBS. Finally, according to the kit from Beyotime Institute of Biotechnology (Shanghai, China), the ROS level was determined in a microplate reader with an excitation wavelength of $485 \mathrm{~nm}$ and an emission wavelength of $535 \mathrm{~nm}$.

2.5. Preparation and Examination of Erythrocytes for Electron Microscope. Erythrocytes were first centrifuged at $2500 \mathrm{rpm}$ 
for $10 \mathrm{~min}$ at $4^{\circ} \mathrm{C}$. The pelleted cells were then mixed with precooled phosphate buffer solution (PBS, $\mathrm{pH} 7.4,5 \mathrm{mM}$ $\mathrm{Na}_{2} \mathrm{HPO}_{4}, 0.1 \mathrm{mM}$ PMSF, and $\left.\mathrm{pH} 8.0\right)$ at ratio $1: 30(\mathrm{v} / \mathrm{v})$ and incubated at $4^{\circ} \mathrm{C}$ for $1 \mathrm{~h}$. The mixture was centrifuged at $4^{\circ} \mathrm{C}, 15000 \mathrm{rpm}$ for $20 \mathrm{~min}$. The pellet was washed three times with cold PBS and centrifugated at the end of each wash at $4^{\circ} \mathrm{C}, 19000 \mathrm{rpm}$ for $20 \mathrm{~min}$. The final pellet was resuspended in cold PBS and the protein concentration was measured by BCA protein assay kit from Beyotime Institute of Biotechnology (Shanghai, China). Erythrocyte membrane protein solution was preserved at concentration of $1 \mathrm{mg} / \mathrm{mL}$ in $-80^{\circ} \mathrm{C}[15,16]$.

For examination of erythrocyte membrane surface morphology, erythrocytes from oxidative stress samples were mixed with $2 \%$ glutaraldehyde at ratio $1: 100(\mathrm{v} / \mathrm{v})$ and then fixed overnight. After being washed 3 times with cold PBS, the samples were then placed in an ion sputtering device (Hitachi, Japan) to be sprayed with metal after drying and then photographed using the S-3400N scanning electron microscope (SEM, Hitachi) [17].

For examination of erythrocyte membrane skeleton structure, erythrocytes from oxidative stress samples were mixed with $\mathrm{NaPi}$ solution $(5 \mathrm{mM}, \mathrm{pH} 7.0)$ containing $2.5 \%$ $(\mathrm{W} / \mathrm{V})$ TritonX-100 at 1:4 ratio $(\mathrm{v} / \mathrm{v})$ and then incubated at $0^{\circ} \mathrm{C}$ for $1 \mathrm{~h}$. The mixture was dropped onto a copper grid (200 mesh), washed three times with $\mathrm{dd}_{2} \mathrm{O}$, and exposed to $1 \%$ uranyl acetate for 5-10 s for negative staining. The H-7500 transmission electron microscope (TEM, Hitachi, Japan) was used to observe the erythrocyte membrane skeletal structure after drying with filter paper.

2.6. Statistical Analysis. Statistical evaluations were carried out using Statistical Package for Social Sciences (SPSS for Windows, version 17.0). All values were expressed as the mean \pm standard deviation (SD). Differences between groups were analyzed by Student's $t$-test. For all tests, $p$ values of less than 0.05 were considered significant.

\section{Investigations and Results}

3.1. Erythrocyte Oxidative Stress Models. Erythrocyte oxidative stress was measured by erythrocyte hemolysis and shown in Figure 2. $\mathrm{H}_{2} \mathrm{O}_{2}$ significantly induced oxidative stress in erythrocytes with dose and time-dependent manner. Over $4 \mathrm{~h}$, there was no hemolysis in erythrocytes without $\mathrm{H}_{2} \mathrm{O}_{2}$ treatment. Erythrocytes treated with 100 and $200 \mathrm{mM} \mathrm{H}_{2} \mathrm{O}_{2}$ showed significant hemolysis after $3 \mathrm{~h}$ treatment $(p<0.01)$. However, erythrocytes treated with 300,400 , and $500 \mathrm{mM}$ $\mathrm{H}_{2} \mathrm{O}_{2}$ displayed significant hemolysis after $1 \mathrm{~h}$ exposure $(p<$ 0.01 ). Since there was significant increase in hemolysis rate after $1.5 \mathrm{~h}$ treatment of erythrocytes with 400 and $500 \mathrm{mM}$ $\mathrm{H}_{2} \mathrm{O}_{2}(p<0.01)$ and no significant difference of hemolysis rate was observed between 400 and $500 \mathrm{mM} \mathrm{H}_{2} \mathrm{O}_{2}$ treatment for $1.5 \mathrm{~h}$, the $400 \mathrm{mM}$ concentration and $1.5 \mathrm{~h}$ exposure were selected for further investigation of biological effects of orientin and luteolin.

3.2. Effects of Orientin and Luteolin on Hemolysis of Erythrocytes. The effects of orientin and luteolin on hemolysis rate of

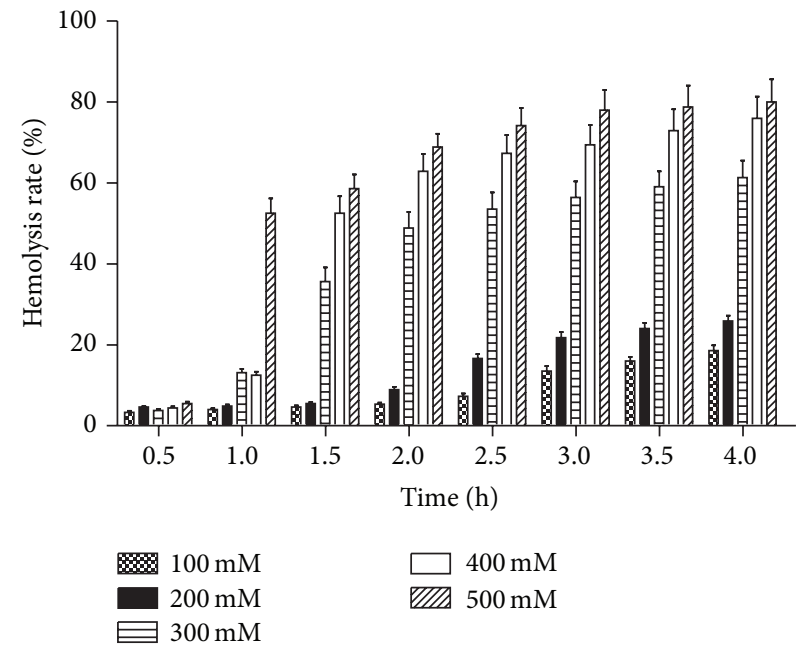

FIGURE 2: Hemolysis of human erythrocytes. It shows hemolysis rate of human erythrocytes induced by different concentrations of $\mathrm{H}_{2} \mathrm{O}_{2}$ at different time intervals. Data are presented as mean \pm SD from six independent experiments.

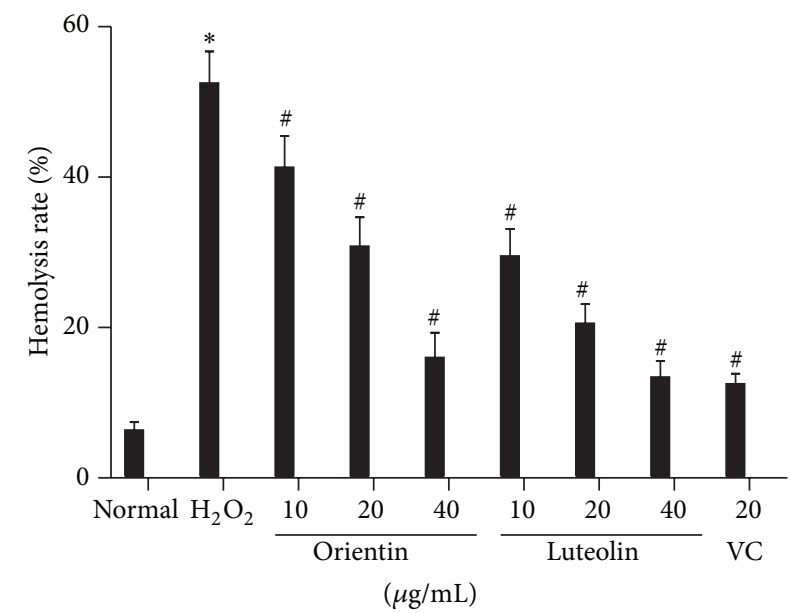

FIGURE 3: Both orientin and luteolin attenuated hemolysis rate of erythrocytes. Oxidative stress induced significant increase in hemolysis rate of erythrocyte. However, both orientin and luteolin ameliorated hemolysis in a dose-dependent manner. Data are presented as mean \pm SD from 10 individual experiments. * represents $p<0.05$ compared to normal control and \# indicates $p<0.05$ compared to oxidative stress erythrocytes.

erythrocytes were shown in Figure 3. Oxidative stress induced significant hemolysis of erythrocytes compared to normal erythrocytes $(p<0.01)$. Both orientin and luteolin significantly attenuated hemolysis of erythrocytes in oxidative stress group in a dose-dependent manner and at the concentrations of orientin (10 or $20 \mu \mathrm{g} / \mathrm{mL})$ were higher than the same concentrations of luteolin $(p<0.05)$. However even with highest concentration of orientin and luteolin $(40 \mu \mathrm{g} / \mathrm{mL})$, hemolysis rates were still higher than normal erythrocytes but at the same level of vitamin C treated erythrocytes.

3.3. Effects of Orientin and Luteolin on Antioxidant Enzymes, ATPase, ROS, and MDA of Human Erythrocyte Exposed to $\mathrm{H}_{2} \mathrm{O}_{2}$. When erythrocytes were treated with $\mathrm{H}_{2} \mathrm{O}_{2}$, there 

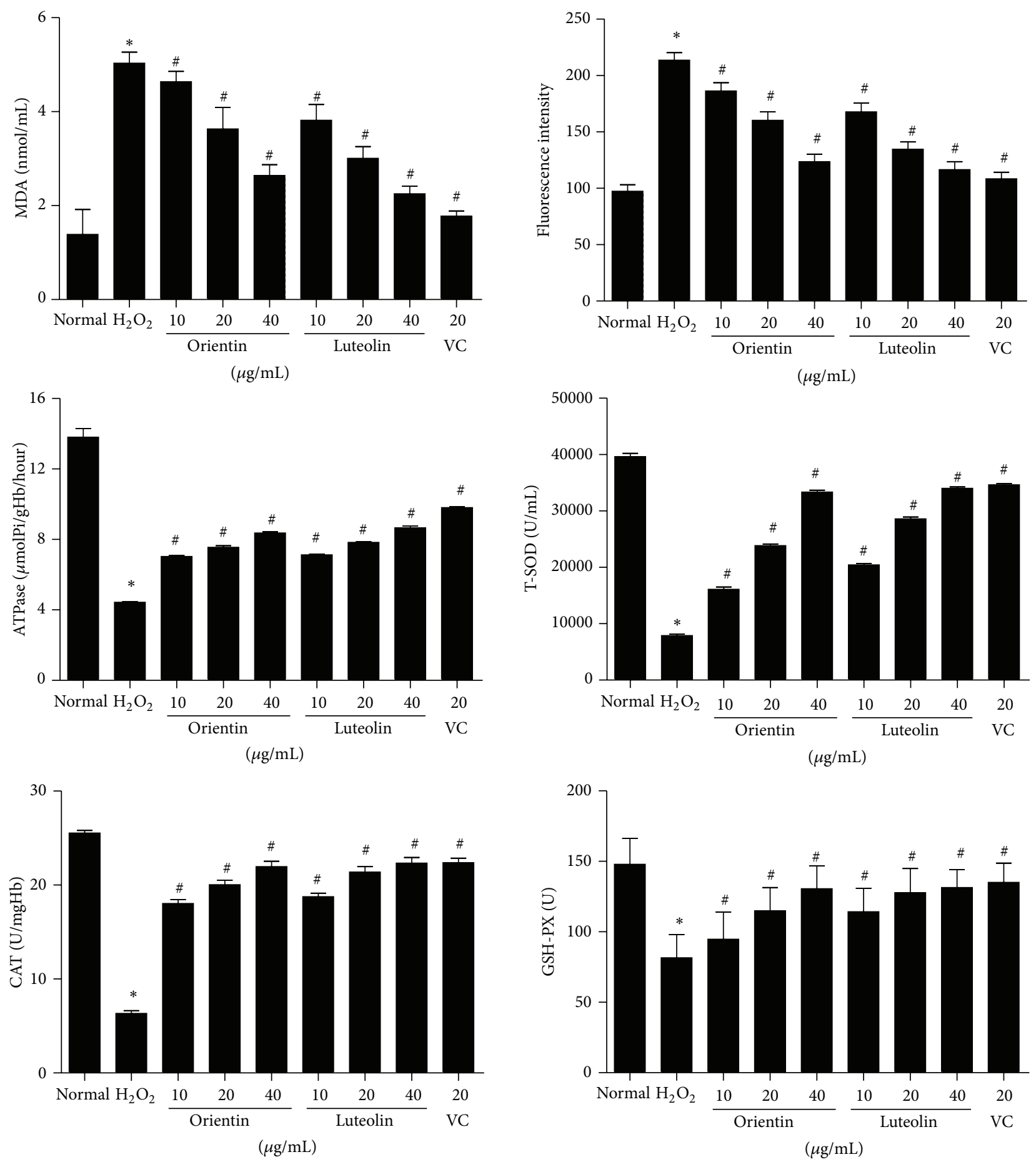

FIgURE 4: Regulations of MDA, ROS, ATPase, total SOD, CAT, and GSH-PX in human erythrocytes under oxidative stress by orientin and luteolin. The panels represent the results of MDA, ROS, ATPase, total SOD, CAT, and GSH-PX with treatment of orientin and luteolin under oxidative stress, respectively. Data are presented as mean \pm SD from 10 individual experiments. * represents $p<0.05$ compared to normal control and \# indicates $p<0.05$ compared to oxidative stress erythrocytes.

were significant increases in ROS and MDA content (Figure 3). However, when erythrocytes were incubated with either orientin or luteolin with $\mathrm{H}_{2} \mathrm{O}_{2}$, there were gradual decreases in both ROS and MDA content in a dosedependent manner and at the concentrations of orientin (10 or $20 \mu \mathrm{g} / \mathrm{mL}$ ) that were higher than the same concentrations of luteolin $(p<0.05)$. Even with highest concentration of either orientin or luteolin, they could not reduce ROS and MDA content to normal erythrocyte levels. Moreover, $\mathrm{H}_{2} \mathrm{O}_{2}$ protected antioxidative enzymes (SOD, CAT, and GSH) and ATPase in erythrocytes and both orientin and luteolin could recover these enzymes activities to almost the levels of $\mathrm{VC}$ 

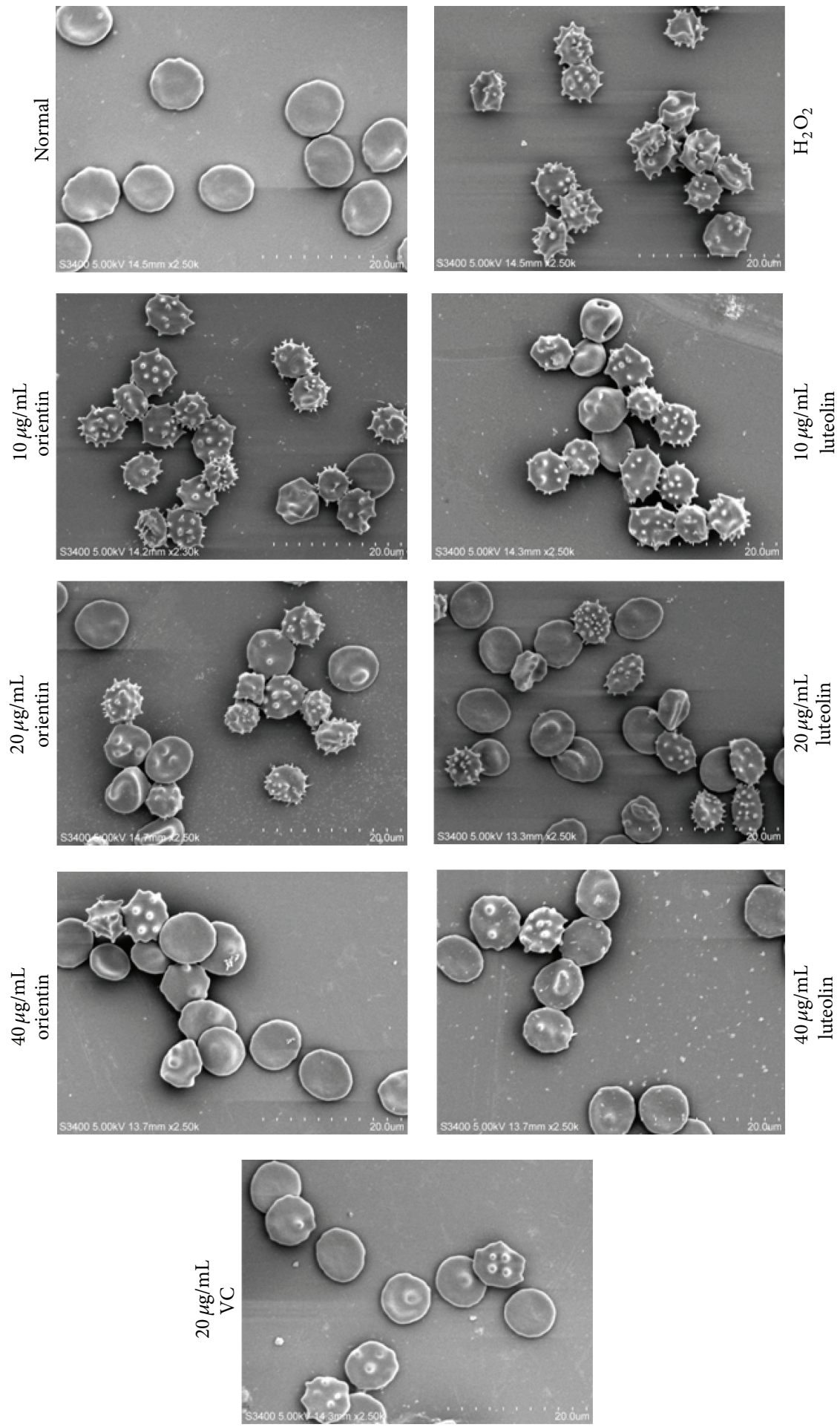

FIGURE 5: Effect of oxidative stress with treatment of orientin and luteolin on erythrocyte cell surface structure. Typical erythrocyte cell surface morphologies were observed under SEM with magnification of 2500x. The treatments were indicated in the figure. The bar in the picture represents $20 \mu \mathrm{m}$.

treated erythrocytes and at the concentrations of orientin $(10$ or $20 \mu \mathrm{g} / \mathrm{mL}$ ) were lower than the same concentrations of luteolin $(p<0.05)$ (Figure 4).

3.4. Effects of Orientin and Luteolin on Surface Morphology and Skeleton Structure of Erythrocytes. Normal erythrocyte surface was smooth and there were no spike-like processes extending out from surface. However when erythrocytes were exposed to $\mathrm{H}_{2} \mathrm{O}_{2}$ erythrocytes, significant amounts of spikelike processes were extended out from the surface. When these erythrocytes were treated with $20 \mu \mathrm{g} / \mathrm{mL}$ of either orientin or luteolin, the amounts of spike-like processes were 


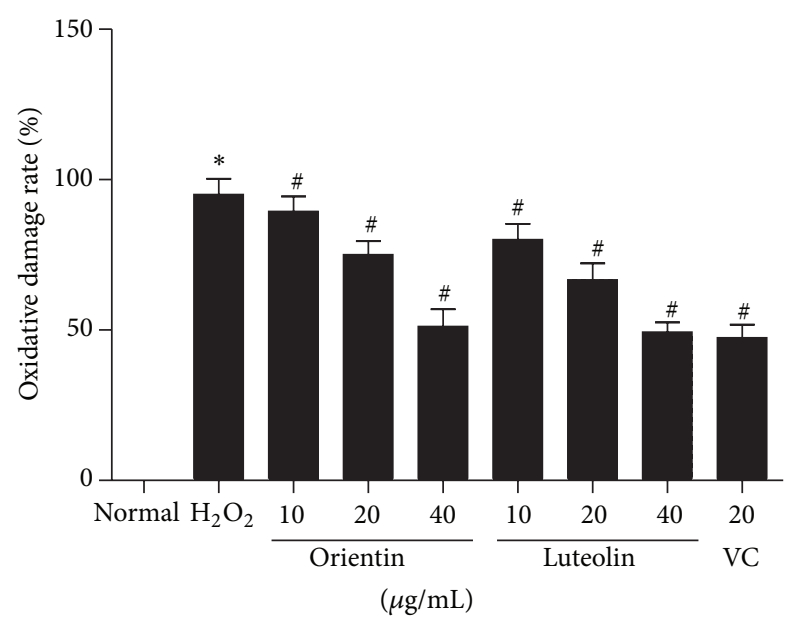

FIGURE 6: Both orientin and luteolin attenuated oxidative damage rate of erythrocytes. Oxidative stress induced significant increase in oxidative damage rate of erythrocyte. However, both orientin and luteolin ameliorated oxidative damage in a dose-dependent manner. Data are presented as mean \pm SD from 10 individual experiments. * represents $p<0.05$ compared to normal control and \# indicates $p<0.05$ compared to oxidative stress erythrocytes.

significantly reduced (Figures 5 and 6). Vitamin C served as positive control and had the same effect on erythrocyte surface morphology as orientin or luteolin. In addition, when erythrocytes were treated with $\mathrm{H}_{2} \mathrm{O}_{2}$, no network-like structure appeared. The J point was not clear and the spectrin tetramer (SP4) assembly was fractured. After treatment with $20 \mu \mathrm{g} / \mathrm{mL}$ of either orientin or luteolin, the erythrocyte membrane skeletal J point was gradually restored, and the SP4 assembly gradually became complete (Figure 7). The same concentration of vitamin C treatment had the same effect on erythrocyte skeletal structure.

\section{Discussion}

Human erythrocytes only have certain lifespan in the blood because they do not have nuclei and mitochondria. Moreover, erythrocytes were exposed to large amount of free radicals that are circulated in the blood. Therefore, oxidative stress of erythrocytes has been proposed as one of the aging mechanism of human erythrocytes [18]. $\mathrm{H}_{2} \mathrm{O}_{2}$ is a free radical and has been widely used to induce oxidative stress in vitro cell experiment. $\mathrm{H}_{2} \mathrm{O}_{2}$ can easily pass through the cell membrane and form highly reactive free radicals with iron molecule by the Fenton reaction [19], which leads to structural and functional damage of the normal cells. This study showed that erythrocyte hemolysis rate of $400 \mathrm{mM} \mathrm{H}_{2} \mathrm{O}_{2}$ at the duration of $1.5 \mathrm{~h}$ was $52.51 \pm 4.22 \%$, reaching the half damage and having no significant difference with $500 \mathrm{mM} \mathrm{H}_{2} \mathrm{O}_{2}$, which was thus selected for further research. Since erythrocytes lack the nuclei and mitochondria, erythrocytes have cellular membrane deformation, suspension instability, and osmotic fragility. Therefore, its function and structural integrity are sensitive to changes of endogenous and exogenous reactive oxygen content. Our results are consistent with the proposal that oxidative stress causes erythrocyte hemolysis.
From oxidative stress hypothesis, any chemicals that can reduce oxidative stress would have ability to reduce erythrocyte injury and prolong its lifespan in the blood. Shortage of erythrocyte lifespan has been demonstrated to associate with anemia, which is a life threaten condition [20]. Several natural products from the traditional Chinese medicine or native medicine are used commonly to treat anemia with excellent outcomes. For example, one study demonstrated the protective effects of quercetin against $\mathrm{H}_{2} \mathrm{O}_{2}$-induced oxidative damage of erythrocytes. Although they showed similar antioxidative activity of quercetin, the erythrocyte hemolysis rate and MDA content are not consistent with the findings in the current study [13]. These differences could be due to the concentrations and duration of used $\mathrm{H}_{2} \mathrm{O}_{2}$. Moreover, the extract of Panax japonicus was also able to attenuate hemolysis of erythrocytes through inhibition of lipid peroxidation of erythrocytes [21, 22]. Furthermore, vitexin was documented to prevent erythrocytes from oxidative damage induced by ROS, preserve antioxidant enzyme activity, and maintain the integrity of erythrocyte morphology and membrane skeletal ultrastructure [23]. Therefore, other flavonoids such as orientin and luteolin may also have similar antioxidative activity.

Orientin belongs to the family of flavonoid glycosides [9] and has abilities to reduce oxidative stress and apoptosis as well as decrease fat and blood sugar $[24,25]$. In ischemicreperfusion model of heart, orientin was able to protect ischemic injury of cardiomyocytes [12] and inhibited apoptosis of these cells [10]. This is consistent with our findings that orientin had antiapoptosis activity in ischemia-reperfusion injury by neutralizing superoxide anions and hydroxyl radicals in vitro [26]. Luteolin belongs to the flavonoid aglycone class [27] and has various activities such as antioxidative, antiinflammatory, and anticancer activities [28-30]. Especially for its antioxidative activity, luteolin glycosides could prevent myocardial cell injury from $\mathrm{H}_{2} \mathrm{O}_{2}$-induced oxidative stress through increasing SOD activity and decreasing the amount of MDA and ROS [11].

Although orientin and luteolin have similar structure of a 15-carbon skeleton, which consists of two phenyl rings (A and $\mathrm{B}$ ) and a heterocyclic ring exception of a C-glycosides linked to glucose of the A ring, our findings indicate that luteolin have better antioxidative activities than orientin in 10 and $20 \mu \mathrm{g} / \mathrm{mL}$ dose groups, which could be related to structural differences between these two compounds. However whether these differences are related to the Cglycoside linked to glucose of the A ring remains to be investigated.

\section{Conclusion}

Both orientin and luteolin could attenuate oxidative stress of erythrocytes through the decrease of the level of ROS and the radicals generation, thereby protecting the antioxidative enzymes and then reducing the damage to structure of cell membrane in $\mathrm{H}_{2} \mathrm{O}_{2}$ induced oxidative stress model of erythrocytes. 

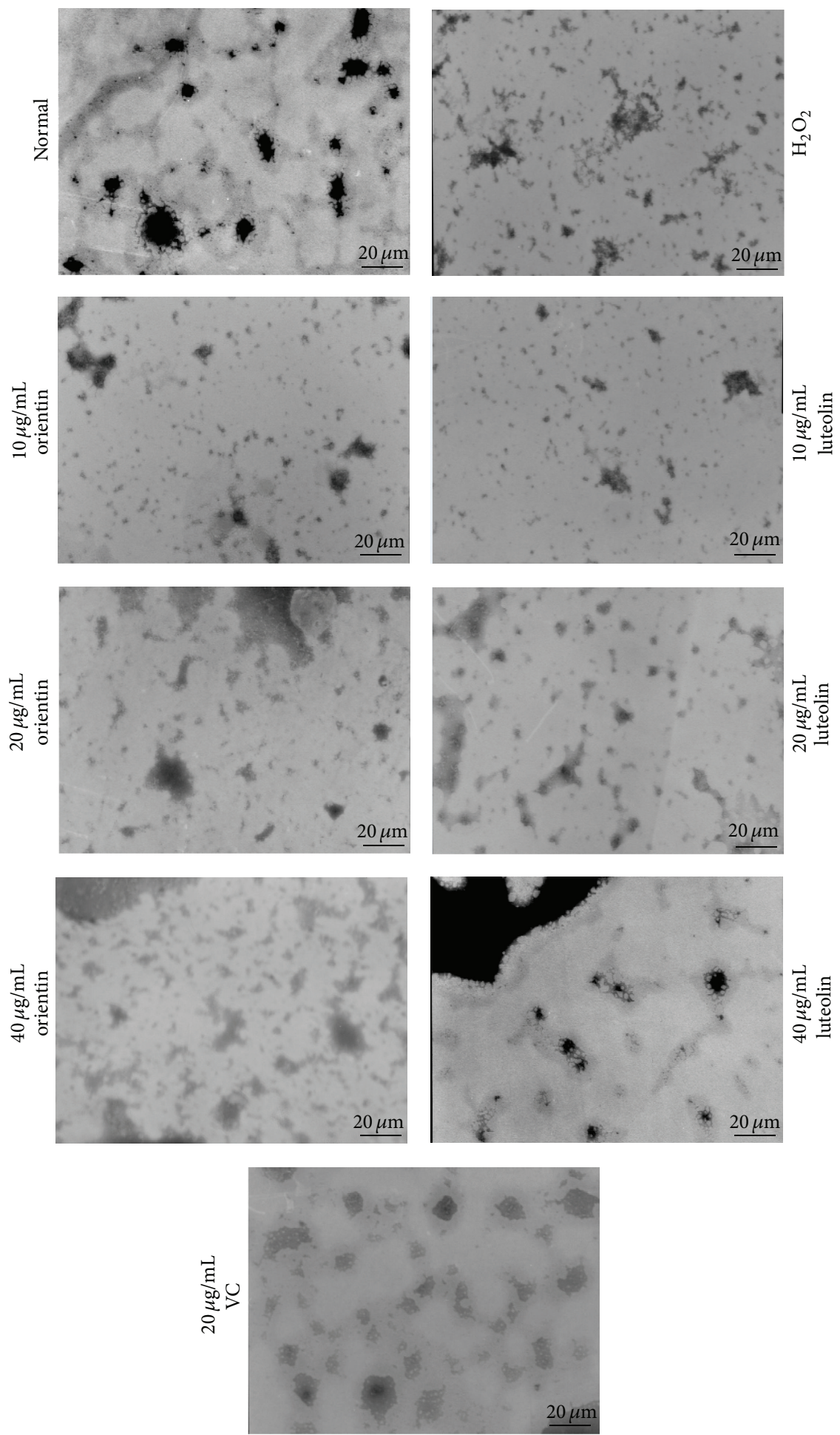

FIGURE 7: Effect of oxidative stress with treatment of orientin and luteolin on erythrocyte cellular structure. Typical erythrocyte cellular structures were observed under TEM with magnification of 30000x. The treatments were indicated in the figure. The bar in the picture represents $20 \mu \mathrm{m}$.

\section{Conflict of Interests}

The authors have declared that there is no conflict of interests.

\section{Authors' Contribution}

Fang An and Shulin Wang contributed equally to this work. 


\section{Acknowledgments}

This work was financially supported by major scientific projects of Hebei North University (ZD1314) and partly supported by the Technology Bureau of Zhangjiakou City (11110015D).

\section{References}

[1] Y. L. Xiong, Effect and Mechanism of Exhaustive Exercise Induced Oxidative Stress in Erythrocytes of Rat, Chongqing University, Chongqing, China, 2014.

[2] S. F. Libregts, L. Gutiérrez, A. M. de Bruin et al., "Chronic IFN- $\gamma$ production in mice induces anemia by reducing erythrocyte life span and inhibiting erythropoiesis through an IRF-1/PU.1 axis," Blood, vol. 118, no. 9, pp. 2578-2588, 2011.

[3] X. M. Sun, The Damage of Human Erythrocyte Membrane Induced by Cigarette Smoke Extract and Inhibited with Tea Catechin, Shaanxi Normal University, Xi’an, China, 2007.

[4] H. L. Wang, Hematology and Hematology Tests, People's Medical Publishing House, Beijing, China, 1997.

[5] R. De Sanctis, R. De Bellis, C. Scesa, U. Mancini, L. Cucchiarini, and M. Dachà, "In vitro protective effect of Rhodiola rosea extract against hypochlorous acid-induced oxidative damage in human erythrocytes," BioFactors, vol. 20, no. 3, pp. 147-159, 2004.

[6] Q. Wang, L. H. Chang, and H. M. Tang, "Extraction of the flavonoids and its research progress in biological activity," Journal of Hebei United University (Natural Science Edition), vol. 33, no. 1, p. 110, 2011.

[7] J. L. Petrick, S. E. Steck, P. T. Bradshaw et al., "Dietary intake of flavonoids and oesophageal and gastric cancer: incidence and survival in the United States of America (USA)," British Journal of Cancer, vol. 112, pp. 1291-1300, 2015.

[8] B. Zhang, The Protective Effects of Anthocyanins from Red Bayberry to Islet Cells from Oxidative Injury and Its Mechanism, Zhejiang University, Zhejiang, China, 2010.

[9] J. Yan, C. H. Qu, J. M. Tian et al., "Separation and purification of orientin and vitexin in Trollius chinensis Bunge by PHPLC," Chinese Traditional Patent Medicine, vol. 33, no. 4, pp. 655-658, 2011.

[10] X.-C. Fu, M.-W. Wang, S.-P. Li, and H.-L. Wang, "Anti-apoptotic effect and the mechanism of orientin on ischaemic/reperfused myocardium," Journal of Asian Natural Products Research, vol. 8, no. 3, pp. 265-272, 2006.

[11] Y. L. Mou, Z. L. Hu, L. Zhou et al., "Protective effects of luteolin-7-O- $\beta$-D-glucoside on neonatal rat myocardial cell injury induced by $\mathrm{H}_{2} \mathrm{O}_{2}$," Journal of Shandong University of Traditional Chinese Medicine, vol. 33, no. 1, pp. 63-65, 2009.

[12] D. N. Liu, Evaluation of Effects of Orientin on Myocardial Ischemia and Its Mechanism, Central University for Nationalities, Beijing, China, 2011.

[13] H. Liu M, H. Ding, and G. Hou, "Quercetin for inhibition of human erythrocyte from peroxidation injury induced by hydrogen peroxide," Journal of Guangdong Medical College, vol. 21, no. 4, pp. 317-318, 2003.

[14] W. Qin, "Study on the inhibitory effect of Rheum on the human erythrocyte hemolysis," Medical Information, vol. 24, no. 7, p. 4532, 2011.

[15] F. Fang and H. Z. Pan, "Preparation and morphological observation of erythrocytes membrane cytoskeletal proteins," Progress in Biochemistry and Biophysics, vol. 20, no. 5, pp. 397-398, 1993.
[16] J. Richard, Investigation into the Membrane Alteration Relevant to the Mechanism of Thermohaemolysis Proteins and Proteomics: A Laboratory Manual, Science Press, Beijing, China, 2003.

[17] X. J. Fan, M. X. Chen, Y. C. Liu et al., "The rapid production of erythrocytes by scanning electron microscopy," Journal of Chinese Electron Microscopy Society, vol. 24, no. 4, pp. 438-438, 2005.

[18] P. Kumar and P. K. Maurya, "Epigallocatechin-3-gallate protects erythrocyte $\mathrm{Ca} 2+-\mathrm{ATP} a \mathrm{se}$ and $\mathrm{Na}+\mathrm{K}+$-ATPase against oxidative induced damage during aging in humans," Advanced Pharmaceutical Bulletin, vol. 4, no. 1, pp. 443-447, 2014.

[19] K. A. Arbos, L. M. Claro, L. Borges, C. A. M. Santos, and A. M. Weffort-Santos, "Human erythrocytes as a system for evaluating the antioxidant capacity of vegetable extracts," Nutrition Research, vol. 28, no. 7, pp. 457-463, 2008.

[20] F. Pourfarzad, M. von Lindern, A. Azarkeivan et al., "Hydroxyurea responsiveness in $\beta$-thalassemic patients is determined by the stress response adaptation of erythroid progenitors and their differentiation propensity," Haematologica, vol. 98, no. 5, pp. 696-704, 2013.

[21] H. B. He, Y. Q. Xu, and N. Wei, “The protection of Japanese ginseng total saponins in $\mathrm{H}_{2} \mathrm{O}_{2}$ induced rats myocardial cell oxidative stress injury," Journal of Chinese Experimental Formulas of Chinese Medicine, vol. 17, no. 17, pp. 187-191, 2012.

[22] J. A. Xu, "Protection of extract of Panax japonicus on oxidative injured erythrocytes," Psychologist Magazine, no. 216, pp. 121122, 2012.

[23] H. Q. Qu, Protective Effects of Vitexin of Trollions on Oxidative Damaged Erythrocyte, Hebei North University, Zhangjiakou, China, 2013.

[24] Y. P. Lin, T. Y. Chen, H. W. Tseng et al., "Neural cell protective compounds isolated from Phoenix hanceana var. formosana," Phytochemistry, vol. 70, no. 9, pp. 1173-1181, 2009.

[25] M. D. P. Nicasio-Torres, J. C. Erazo-Gómez, and F. CruzSosa, "In vitro propagation of two antidiabetic species known as guarumbo: Cecropia obtusifolia and Cecropia peltata," Acta Physiologiae Plantarum, vol. 31, no. 5, pp. 905-914, 2009.

[26] G. D. Yang, N. Rao, J. M. Tian et al., "Studies on outside antioxidation of orientin and vitexin of Trollius chinensis Bunge," Lishizhen Medicine and Materia Medica Research, vol. 22, no. 9, pp. 2172-2173, 2011.

[27] H. Yao, Z. Shang, P. Wang et al., "Protection of Luteolin7-O-glucoside against doxorubicin-induced injury through PTEN/Akt and ERK pathway in H9c2 cells," Cardiovascular Toxicology, 2015.

[28] C. Bumke-Vogt, M. A. Osterhoff, A. Borchert et al., "The flavones apigenin and luteolin induce FOXO1 translocation but inhibit gluconeogenic and lipogenic gene expression in human cells," PLoS ONE, vol. 9, no. 8, Article ID e104321, 2014.

[29] L. Liu, Z. Peng, Z. Xu, and X. Wei, "Effect of luteolin and apigenin on the expression of Oct-4, Sox2, and c-Myc in dental pulp cells with in vitro culture," BioMed Research International, vol. 2015, Article ID 534952, 10 pages, 2015.

[30] M. T. Yasuda, K. Fujita, T. Hosoya, S. Imai, and K. Shimoi, "Absorption and metabolism of luteolin and its glycosides from the extract of chrysanthemum morifolium flowers in rats and caco-2 cells," Journal of Agricultural and Food Chemistry, vol. 63, no. 35, pp. 7693-7699, 2015. 


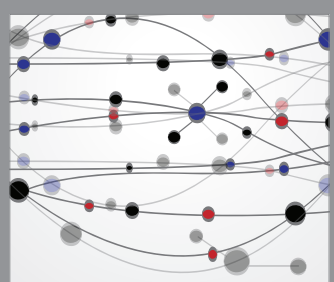

The Scientific World Journal
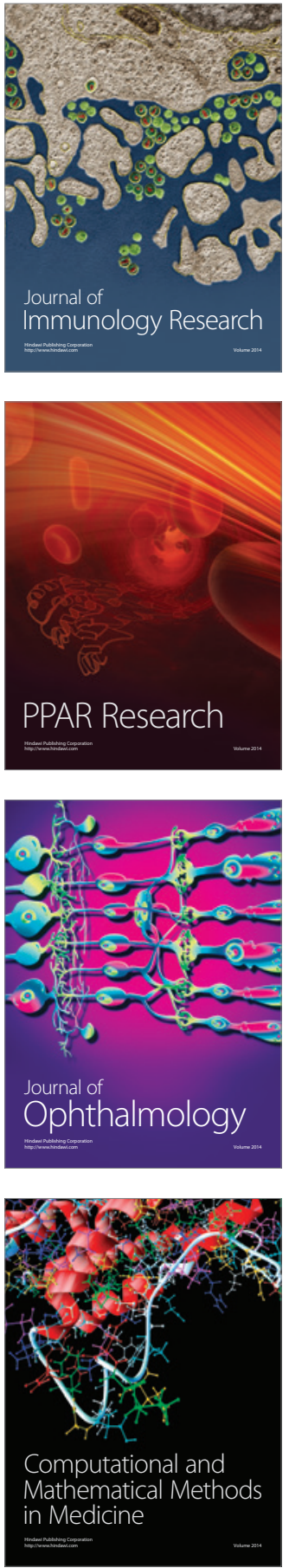

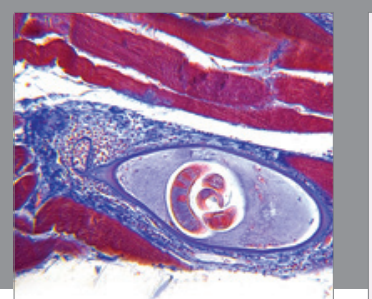

Gastroenterology Research and Practice

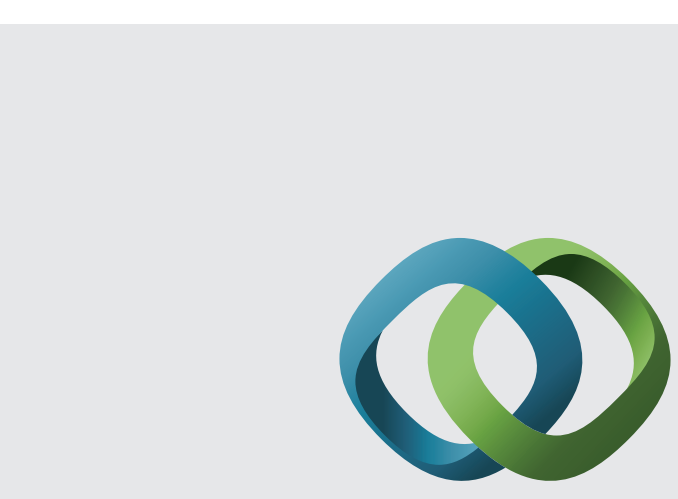

\section{Hindawi}

Submit your manuscripts at

http://www.hindawi.com
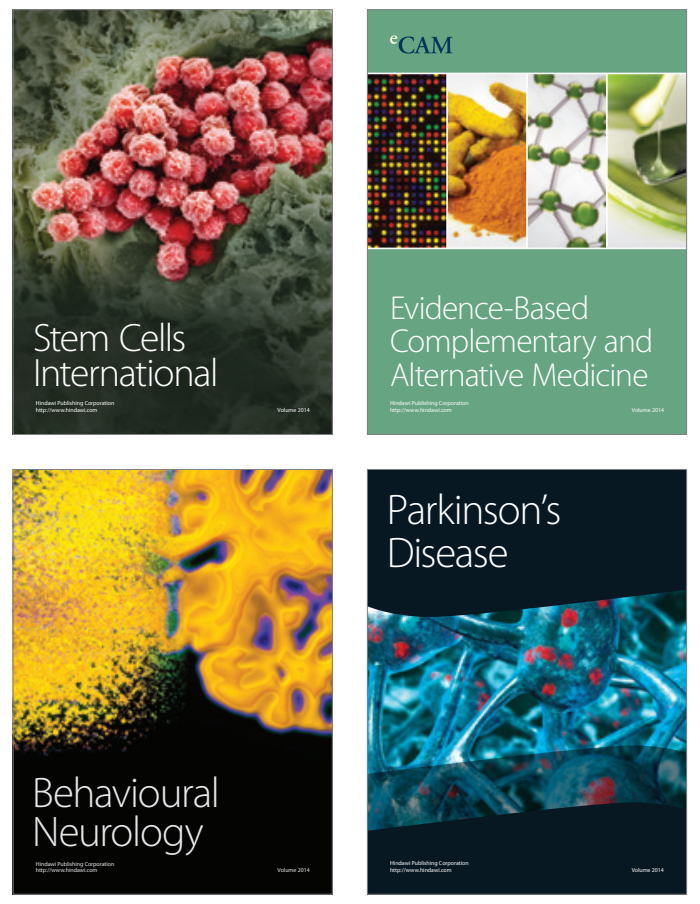
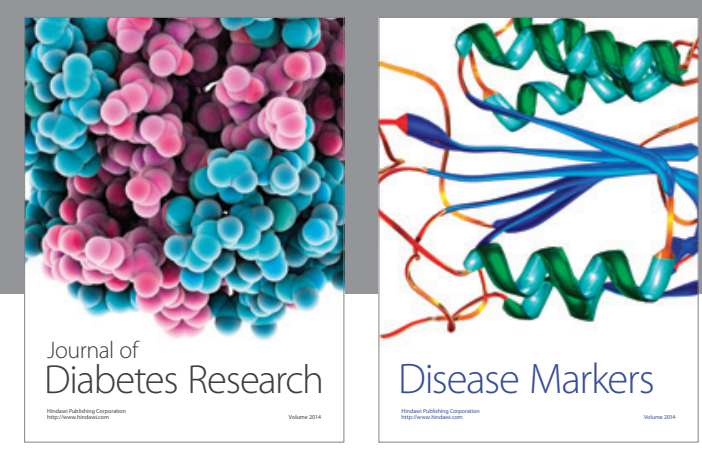

Disease Markers
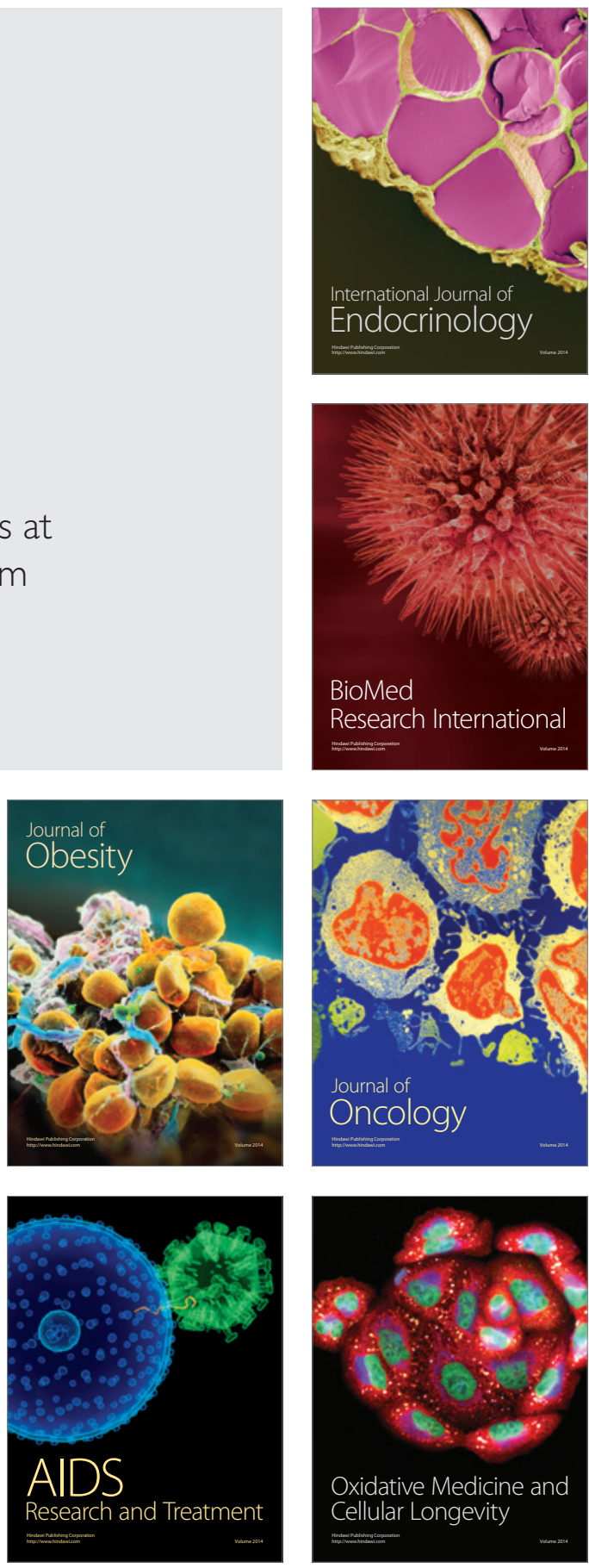